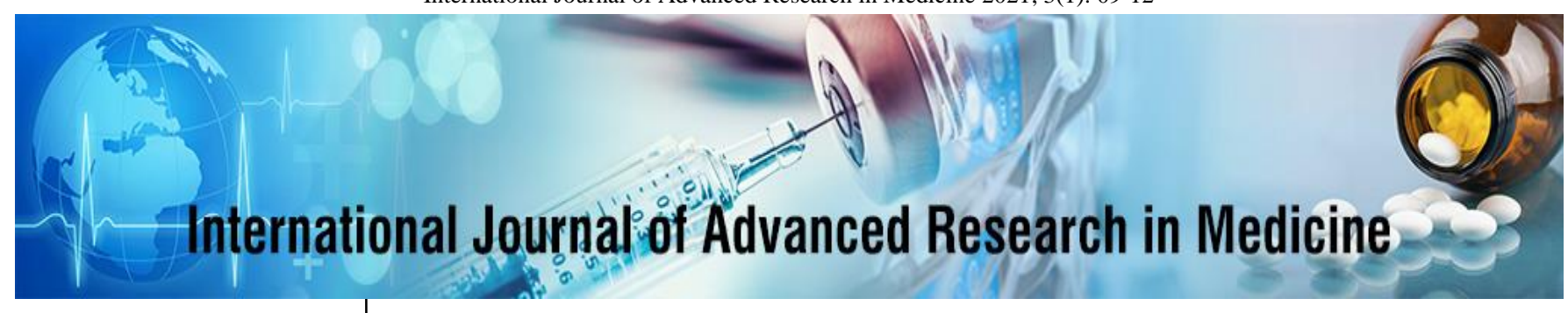

E-ISSN: 2706-9575

P-ISSN: 2706-9567

IJARM 2021; 3(1): 09-12

Received: 09-11-2020

Accepted: 13-12-2020

Dr. Mohammed Ismail KK Assistant Professor, Department of General

Medicine, P K Das Institute of Medical Sciences,

Vaniyamkulam, Ottapalam,

Palakkad, Kerala, India

\section{Dr. Riyas C}

Assistant Professor,

Department of General

Medicine, P K Das Institute of

Medical Sciences,

Vaniyamkulam, Ottapalam,

Palakkad, Kerala, India
Corresponding Author: Dr. Riyas C

Assistant Professor,

Department of General

Medicine, P K Das Institute of

Medical Sciences,

Vaniyamkulam, Ottapalam,

Palakkad, Kerala, India

\title{
Prevalence of insulin resistance among patients with cirrhosis of liver
}

Dr. Mohammed Ismail KK and Dr. Riyas C

DOI: https://doi.org/10.22271/27069567.2021.v3.i1a.88

\section{Abstract}

Introduction: Liver cirrhosis is an unique clinical entity in which long-term diabetes mellitus (DM) can predispose individuals to cirrhosis and, on the other hand, long-term cirrhosis of the liver can predispose cirrhosis to DM, secondary to glucose and insulin metabolism.

Aims: The aim of this study is to determine the prevalence of insulin resistance in liver cirrhosis.

Materials and Methods: This cross sectional case control study conducted in department of medicine in 80 patients for duration of 1 year. All cirrhotic patients irrespective of aetiology were subjected to fasting plasma glucose level and fasting plasma insulin levels and insulin resistance was calculated by HOMA-IR method. Study was statistically analysed.

Results: Insulin resistance was found in 62 cases with IR in ratio in $<1.64$ is $0.81+0.5$ and IR ratio $>1.64$ is $4.6+2.9$. with $\mathrm{p}$ value is $<0.001$ which is highly significant. Insulin Resistance among alcoholic, NAFLD, Autoimmune hepatitis and HCC patients was found significant when compared to non-insulin resistant patients. Prevalence of Insulin resistance increased with increasing grades of child Pugh score. As Insulin Resistance was detected in 62 out of 80 patients who even had $\mathrm{HbA1c}$ levels below 5.7 and in 28 out of 62 had FPG levels below 100 .

Conclusion: Insulin resistance may be used as a valuable prognosis marker in patients with cirrhosis of the liver, serum insulin levels can be prescribed as routine tests in these patients. Diabetes mellitus raises the risk of $\mathrm{HCC}$ in patients with cirrhosis. Thus, through early detection of diabetes mellitus, by measuring insulin resistance using the HOMA-IR process, we can prevent disease progression.

Keywords: hepatogenic diabetes, cirrhosis, insulin resistance

\section{Introduction}

Diabetes mellitus (DM) is a complex metabolic clinical entity associated with a high risk of micro-and macro-vascular complications, poor quality of life and shortened life expectancy. There are at least 170 million people worldwide suffering from DM, and this is expected to affect almost 266 million by 2030. In India, the prevalence is approximately 10\%, i.e. 31 million people are likely to be diabetic, and this number is likely to cross 80 million by 2030 . Studies have shown that Indians are predisposed to DM at a much younger age with a higher risk of DM-related complications due to a lower level of obesity ${ }^{[1,2]}$.

The association between diabetes mellitus and cirrhosis of the liver has shown increased insulin resistance (IR). Actually, it is uncertain if type 2 diabetes mellitus, in the absence of other risk factors leading to metabolic syndrome (obesity and hypertriglyceridemia), may be a risk factor for the development and progression of liver disease. On the other hand, diabetes, which occurs as a cirrhosis complication, is classified as "hepatogenic diabetes" and is not recognised as a separate autonomous body by the American Diabetes Association and the World Health Organization. IR is defined where a normal or elevated level of insulin produces an attenuated biological response ${ }^{[3]}$.

Compensatory hyperinsulinemia occurs when pancreatic $\beta$-cell secretion rises to maintain normal blood glucose levels in peripheral IR settings in muscle and adipose tissues. IR results from defects either at the receptor level or in the insulin receptor substrate molecules. Hyperinsulinemia has also had several adverse effects on the vascular bed.

Patients with cirrhosis and insulin resistance need daily glycaemic control as they may develop impaired glucose tolerance and diabetes in the future, which have definite clinical consequences in the form of reduced response to care, rapid progression to fibrosis, risk of hepatocellular carcinoma and increased cirrhosis complications ${ }^{[4]}$. 
HOMA is a glucose-insulin dynamics relationship model that predicts fast steady-state glucose and insulin concentrations for a wide range of potential IR-and $\beta$-cellfunction combinations. The HOMA model has been validated and commonly used in clinical data to assess the degree of IR ${ }^{[5]}$.

HOMA-IR clearly predicts the development of type 2 diabetes, and glucose tolerance status. The HOMA-IR value of more than 1.64 indicated abnormally high IR. This study was conducted to study the prevalence of insulin resistance in liver cirrhosis.

\section{Materials and Methods}

This cross-sectional case control study conducted in department of medicine in 80 patients for duration of 1 year from May 2018 to April 2019 after getting informed consent from all the patients involved in this study.

Inclusion criteria Patients with cirrhosis already proven by imaging, who was regularly attending Medicine and outpatient clinic and inpatients in medical ward were included. Exclusion criteria: HbA1c $>6.5$, Fasting plasma glucose $\geq 126 \mathrm{mg} / \mathrm{dl}$, Patients receiving insulin and drugs like oral hypoglycaemics, corticosteroids, Thiazides and Phenytoin and Pregnant women.

All the patient have been subjected to necessary investigations and Modified child Pugh scoring was done.

The procedures to be employed were explained to the patients, and written consent was obtained from all patients included in the study. A detailed history was taken, and a thorough clinical examination was done followed by further investigations, all of which were recorded in a pre-designed, structured proforma. Height and weight were measured. Presence of ascites and pedal oedema were noted.

Child Pugh classification of cirrhosis: The Child Pugh score was originally developed by Child and Turcotte. Child Pugh Scores were calculated by adding the scores of the five factors (serum bilirubin, serum albumin, prothrombin time, grade of ascites and hepatic encephalopathy) in order to classify patients according to severity of CLD into Child Pugh class A (a score of 5-6), B (7-9), or C (10 or above). Body mass index was calculated using the formula weight (kg)/ height* height (m). If ascites was present, then correction of 4 was given for weight.

Insulin resistance was taken by HOMA $1 \mathrm{R}$ calculation.

\{fasting insulin (ц U/dl) * fasting glucose $(\mathrm{mmol} / \mathrm{L})\} / 22.5$.

A value $>2.7$ is taken as insulin resistance.

The study required 80 cases with the assumption that DM was likely to be prevalent in $15 \%$ of cirrhotic patients. error of $8 \%$.

Data were analysed using the IBM-SPSS version 20.0 application. Chi-square test or proportion tests were used for comparison of percentages, Linear regression analysis and logistic regression analysis were done. $P<0.05$ was considered significant.

\section{Results}

The age of all patients included in this study varied between 24-75 years. The most common age group studied was

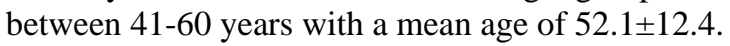

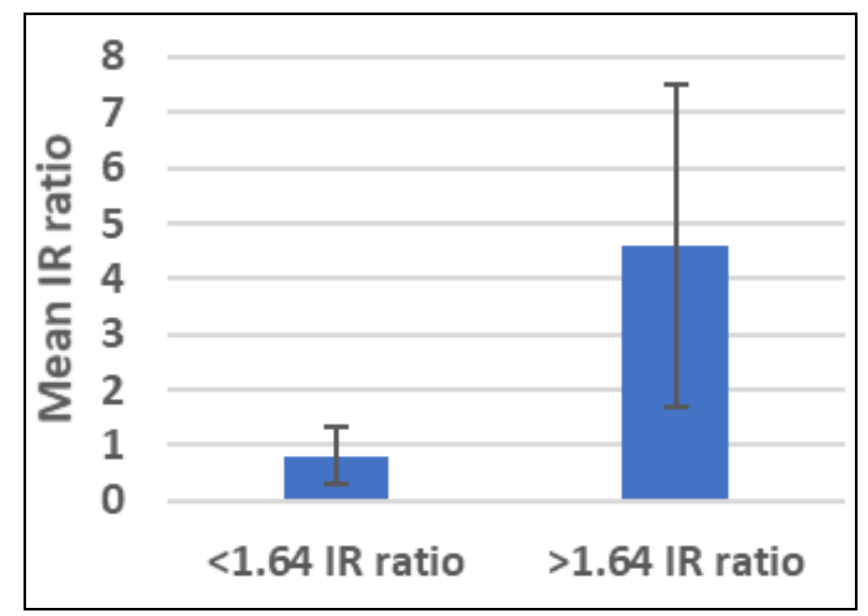

Fig 1: Insulin resistance in cirrhosis patients

Insulin resistance was found in 62 cases and 18 cases are without insulin resistance.
Mean IR in ratio in $<1.64$ is $0.81+0.5$ and IR ratio $>1.64$ is $4.6+2.9$. with $\mathrm{p}$ value is $<0.001$ which is highly significant.

Table 1: Insulin resistance in different parameters.

\begin{tabular}{|c|c|c|c|}
\hline Variables & IR ratio >1.64 (n=62) in number of cases & IR ratio <1.64 (n=18) in Number of cases & P-Value \\
\hline \multicolumn{2}{|c|}{ Aetiology } & 7 & $0.01^{*}$ \\
\hline Alcohol & 31 & 13 & 0.31 \\
\hline NAFLD & 13 & 4 & $0.01^{*}$ \\
\hline HBV & 21 & 3 & 0.20 \\
\hline HCV & 3 & 0 & $0.01^{*}$ \\
\hline Autoimmune hepatitis & 3 & 3 & $0.05^{*}$ \\
\hline HCC & 7 & & \\
* Significance & \multicolumn{3}{|c|}{}
\end{tabular}

Insulin Resistance among alcoholic, NAFLD, Autoimmune hepatitis and HCC patients was found significant when compared to non-insulin resistant patients. 
Table 2: Child Pugh score in comparison with insulin resistance

\begin{tabular}{|c|c|c|c|c|c|}
\hline Child Pugh score & Total no of patients & Number of cases with IR (>1.64) & percentage & Mean+SD & P-Value \\
\hline 5-6 class A & 7 & 4 & 57 & $5.3+2.6$ & $<0.05^{*}$ \\
\hline 7-9 class B & 39 & 27 & 69 & $6.4+2.9$ & $<0.05^{*}$ \\
\hline$>10$ class C & 44 & 31 & 70.4 & $5.7+2.9$ & $<0.05^{*}$ \\
\hline
\end{tabular}

$70.4 \%$ of Child Pugh Class C had IR with a mean of $5.7+$ 2.9, 69\% of Child Pugh Class B patients had IR and mean was $6.4+2.9$,. and $57 \%$ of child Pugh class A patients had IR with Mean insulin resistance of 5.3 \pm 2.6 .

Prevalence of Insulin resistance increased with increasing grades of child Pugh score. As Insulin Resistance was detected in 62 out of 80 patients who even had $\mathrm{HbA} 1 \mathrm{c}$ levels below 5.7 and in 28 out of 62 had FPG levels below 100 which makes IR a better predictor of impending Diabetes mellitus.

\section{Discussion}

Chronic ALD is mediated by combined effects of IR and toxic injury. Hepatic IR is caused by defects in intracellular signalling, including impairments in receptor binding and receptor tyrosine kinase activation. Ethanol also inhibits tyrosine phosphorylation of insulin receptor substrate proteins, needed to transmit insulin and insulin-like growth factor receptor signals. he also stated that IR was least commonly associated with HBV-related liver disease and HOMA-IR rises only after advancement in the stage of liver disease. Diabetes can lead to non-alcoholic fatty liver disease and non-alcoholic steatohepatitis and ultimately cirrhosis. But conversely cirrhosis can lead to impaired glucose tolerance and diabetes. Diabetes occurring in the setting of cirrhosis is called hepatogenous diabetes. Hepatogenous diabetes has little micro and macrovascular complications

As per the present study insulin resistance was found in 62 out of 80 cases. The $p$ value is $<0.001$ which is highly significant. Studies conducted in Spain by Erice E et al. ${ }^{[6]}$ on-insulin resistance in patients of cirrhosis with portal hypertension also showed that insulin resistance was present in $60 \%$ of his studied population. Goswami et al. ${ }^{[7]}$ study Insulin resistance was present in $68.5 \%$ of euglycemic cirrhotic patients.

Insulin Resistance among alcoholic, NAFLD, Autoimmune hepatitis and HCC patients was found significant when compared to non-insulin resistant patients. Several studies suggest that type 2 diabetes mellitus may have an etiological role in chronic liver disease and HCC regardless of alcohol and viruses. ${ }^{8}$ In a recent case-control study that included 465 patients, diabetes mellitus prevalence was higher in patients with HCC than in controls $(31.2 \%$ vs $12.7 \%$, OR 3.12, 95\% CI: 2.22-4.43). The diabetes mellitus had been diagnosed prior to the occurrence of HCC in $84 \%$ of cases with an average duration of 181.4 months indicating that it was type 2 diabetes mellitus in most cases. But aetiology had not influenced IGT and diabetes according to some study. A study conducted by Bonora, et al. [9] showed inverse correlation between clamp mediated glucose disposal and HOMA estimated insulin sensitivity. They also validated the study for use in large epidemiological purposes

In present study $70.4 \%$ of Child Pugh Class C had IR with a mean of $5.7+2.9,69 \%$ of Child Pugh Class B patients had IR and mean was $6.4+2.9$, and $57 \%$ of child Pugh class A patients had IR with Mean insulin resistance of 5.3 \pm 2.6 .
Present study is also supported by Holstein et al. ${ }^{[10]}$ who did a prospective cohort study on 52 histologically confirmed patients of liver cirrhosis. $44 \%$ of his patients had child Pugh score A, 37\% score B and 19\% score C. Seventy- one percent $(71 \%)$ of his patients with liver cirrhosis had manifest diabetes, $25 \%$ impaired glucose tolerance and only $4 \%$ normal glucose tolerance.

Similar to the present study, Gharvi et al. ${ }^{[11]}$ also found that the prevalence of insulin resistance for groups A, B, and C of Child-Pugh classification were $31.6 \%, 66.7 \%$ and $74.1 \%$ respectively and the relationship between IR and severity of liver cirrhosis was significant statistically $(\mathrm{p}=0.01)$.

In present study Prevalence of Insulin resistance increased with increasing grades of child Pugh score. As Insulin Resistance was detected in 62 out of 80 patients who even had HbA1c levels below 5.7 and in 28 out of 62 had FPG levels below 100 which makes IR a better predictor of impending Diabetes mellitus. Fasting blood sugar and $\mathrm{HbA1C}$ are likely to less informative as cirrhotic patients have a reduced red blood cell liver span and one may get false low $\mathrm{HbA} 1 \mathrm{C}$ levels ${ }^{12,13}$ and reduce the rates of detection of both IGT and NODM. In a recent study by Nishida $e t$ al. ${ }^{[14]}$ involving cirrhotic patients with normal fasting blood glucose levels and without a family history of type $2 \mathrm{DM}$, $77 \%$ had DM or IGT on GTT.

Goswami et al. [7] made similar observations i.e. a significantly high HOMA-IR in recently diagnosed diabetes in cirrhosis patients compared to those without DM i.e. 4.9 $\pm 1.9(P<0.0001)$. Insulin resistance is common in euglycemic cirrhosis and with advancement of liver disease; there is a compensatory increase in pancreatic $\beta$-cell insulin secretion to overcome the insulin resistance. However, despite this over a period of time with a fall in $\beta$-cell function, hepatogenous diabetes is likely to set in.

Pathogenesis, assessment and cause of death in insulin resistance related to liver disease differ from those of lifestyle-related insulin resistance. Furthermore, exogenous insulin or sulfonylureas may be harmful because these agents may promote hepatocarcinogenesis. There is, therefore, a need for a unique therapeutic strategy for hepatogenous insulin resistance.

\section{Conclusion}

However, with prolonged or sustained IR pancreatic $\beta$-cell function loss occurs, which may result in the development of hepatogenous diabetes. So insulin resistance can be used as an important prognostic marker in patients with cirrhosis of liver, serum insulin levels can be recommended as routine investigation in these patients. Moreover, as there can be increase in complications in patients detected to have insulin resistance and diabetes in cirrhosis, early intervention for control of diabetes in such cases may prevent/reduce the occurrence of complications. Insulin resistance developing in cirrhotic patients if detected early can help in preventing overt diabetes. 


\section{References}

1. Chawla A, Chawla R, Jaggi S. Microvasular and macrovascular complications in diabetes mellitus: Distinct or continuum?. Indian J Endocrinol Metab. 2016;20(4):546-551.

2. International Diabetes Federation. IDF Atlas. 7th edition. [Last accessed on 2015 Dec 27]. Available from: http://www.diabetesatlas.org.

3. Megyesi C, Samols E, Marks V. Glucose tolerance and diabetes in chronic liver disease. Lancet 1967;2:10511056.

4. Cersosimo E, Solis-Herrera C, Trautmann ME, Malloy J, Triplitt CL. Assessment of pancreatic $\beta$-cell function: review of methods and clinical applications. Curr Diabetes Rev 2014;10(1):2-42.

doi:10.2174/1573399810666140214093600

5. Wilcox G. Insulin and insulin resistance. Clin Biochem Rev 2005;26(2):19-39.

6. Eva Erice, Elba Llop, Annalisa Berzigotti, Juan G. Abraldes, Ignacio Conget, Susana Seijo. Insulin resistance in patients with cirrhosis and portal hypertension. Am J Physiol Gastrointest Liver Physiol 2012;302(12):G1458-65.

7. Amitava Goswami, Narendra Bhargava, Sunil Dadhich, Ganaraj Kulamarva. Insulin resistance in euglycemic cirrhosis. Annals of Gastroenterology 2014;27:237-243.

8. El-Serag HB, Everhart JE. Diabetes increases the risk of acute hepatic failure. Gastroenterology 2002;122:1822-1828.

9. Bonora E, Formentini G, Calcaterra F, et al. HOMAEstimated Insulin Resistance Is an Independent Predictor of Cardiovascular Disease in Type 2 Diabetic Subjects. Diabetes Care 2002;25:1135-1141.

10. Holstein A, Hinze S, Thiessen E, Plaschke A, Egberts EH. Clinical implications of hepatogenous diabetes in liver cirrhosis. J Gastrenterol Hepatol 2002;17:677-81.

11. Gharavi A, Mohammadi AHA, Ziaee A, Sarookhani MR, Javadi A, Haghazali S, et al. Investigation of insulin resistance in patients with liver cirrhosis and its relationship with severity of disease. J Qazvin Univ Med Sci 2008;12(1):27-34.

12. Nishida T, Tsuji S, Tsujii M, Arimitsu S, Haruna Y, Imano E, et al. Oral glucose tolerance test predicts prognosis of patients with liver cirrhosis. Am J Gastroenterol 2006;101:70-5.

13. Kawaguchi T, Taniguchi E, Itou M, Sakata M, Sumie S, Sata M, et al. Insulin resistance and chronic liver disease. World J Hepatol 2011;3:99-107.

14. Petta S, Cammà C, Di Marco V, Macaluso FS, Maida M, Pizzolanti G, et al. Hepatic steatosis and insulin resistance are associated with severe fibrosis in patients with chronic hepatitis caused by $\mathrm{HBV}$ or $\mathrm{HCV}$ infection. Liver Int 2011;31:507-15. 